\title{
A!
}

This is an electronic reprint of the original article.

This reprint may differ from the original in pagination and typographic detail.

Ponnada, Tushara; Al-Tous, Hanan; Tirkkonen, Olav

\section{Location-Free Beam Prediction in mmWave Systems}

Published in:

Proceedings of IEEE 93rd Vehicular Technology Conference, VTC 2021

DOI:

10.1109/VTC2021-Spring51267.2021.9448938

Published: 15/06/2021

Document Version

Peer reviewed version

Please cite the original version:

Ponnada, T., Al-Tous, H., \& Tirkkonen, O. (2021). Location-Free Beam Prediction in mmWave Systems. In Proceedings of IEEE 93rd Vehicular Technology Conference, VTC 2021 [9448938] (IEEE Vehicular Technology Conference). IEEE. https://doi.org/10.1109/VTC2021-Spring51267.2021.9448938

This material is protected by copyright and other intellectual property rights, and duplication or sale of all or part of any of the repository collections is not permitted, except that material may be duplicated by you for your research use or educational purposes in electronic or print form. You must obtain permission for any other use. Electronic or print copies may not be offered, whether for sale or otherwise to anyone who is not an authorised user. 


\section{Location-Free Beam Prediction in mmWave Systems}

\author{
Tushara Ponnada \\ Dept. of Commun. and Networking \\ Aalto University \\ Espoo, Finland \\ tushara.ponnada@aalto.fi
}

\author{
Hanan Al-Tous \\ Dept. of Commun. and Networking \\ Aalto University \\ Espoo, Finland \\ hanan.al-tous@aalto.fi
}

\author{
Olav Tirkkonen \\ Dept. of Commun. and Networking \\ Aalto University \\ Espoo, Finland \\ olav.tirkkonen@aalto.fi
}

\begin{abstract}
Channel charting is a method for creating radiomaps of a cell that capture the neighborhood relationships between User Equipments (UEs) in the cell based on machine learning techniques. In this paper, we leverage channel charting for predicting the best Base Station (BS) beam to serve a given UE in a massive-MIMO $5 \mathrm{G}$ network. Because of the autonomous beamforming at the UE in 5G networks, the BS cannot determine the best beam for transmission to a UE by measuring the UE transmissions in all the BS beams. To address this issue, we propose a framework to predict the best $\mathrm{BS}$ beam for a mobile UE in the next transmission instant by utilizing the channel charts of the cell that the UE is currently in. We evaluate the prediction accuracy of the framework using simulated channels from QuaDRiGa channel generator. We compare the performance of channel chart and physical location based predictors. While the prediction accuracy attained using channel charting is less than that of the prediction using physical locations, there remain several ways to improve the performance.
\end{abstract}

Index Terms-mmWave, CSI features, 5G TDD system, channel charting, BS beam prediction.

\section{INTRODUCTION}

Millimeter Wave (mmWave) communication is one of the enabling technologies for Beyond Fifth-Generation (B5G) communication systems, providing large bandwidths that can help meet the formidable capacity demands and low latency requirements. However, mmWave transmissions are prone to high propagation loss for non-line-of-sight cases. Large antenna arrays as used in Massive Multiple-Input-MultipleOutput (mMIMO) technology can provide significant beamforming gains and directivity to help overcome the attenuation suffered by mmWave transmissions [1], [2].

Because of the high attenuation of mmWave transmissions, precise alignment of transmission beams towards the receiving UEs is necessary to have reliable communication links. This may be difficult in mMIMO systems with mobile users. Accordingly, mmWave wireless systems may suffer from significant overhead in searching for the optimal beam to serve mobile UEs. If there were only one UE antenna, it would be rather simple for the BS to measure the best beam, once there is a UE transmission. In a Time Division Duplexing (TDD) system with channel reciprocity, the same beam could directly be used for transmission. What makes the thing challenging

This work was funded in part by Huawei Technologies Co., Ltd. is that in $5 \mathrm{G}$ networks the UE autonomously selects a beam direction to transmit to in uplink, and to receive from in downlink. Now this UE beam is not under the control of the $\mathrm{BS}$, and it is unclear what consequences a change of BS beam would have. As a result, the BS cannot simply determine the best BS beam by measuring the UE transmissions in all the BS beams. Hence, the problem of determining the best BS beam gains significance in case of mobile $5 \mathrm{G}$ systems.

To reduce the latency associated with searching for the optimal beam exhaustively, use of Machine Learning (ML) techniques with a data-driven approach has been gaining significance. Optimal beam prediction for vehicles in mmWave networks is performed by training ML models with a richer data set including physical locations of not just the vehicle but also that of its neighbouring vehicles and some side information together termed as situational awareness in [3]. In [4] a ML solution leveraging geolocation side information is considered for enhancing beam management in mmWave wireless systems. Support vector machines are used to model the mapping between the UEs geolocations and their serving beams/cells in a multiuser, multi-cell environment. In [5], ML assisted beam training is performed for mmWave cellular systems using realistic beamforming data and GPS coordinates of UEs.

Channel charting is the application of unsupervised machine learning techniques to process the high-dimensional Channel State Information (CSI) of UEs in a cell, and obtain a logical radio-map which is a lower-dimensional representation of the cell where the neighborhood relationship of the UEs is preserved [6]. Channel charting can be performed for a single BS to obtain what is called a Single-Point Channel Chart (SPCC) or for multiple BSs from their individual channel charts to obtain a Multi-Point Channel Chart (MPCC) [7]. Once Channel Chart (CC) is constructed by collecting sufficiently sampled CSI information from a cell, it facilitates Radio Resource management (RRM) functions like handover prediction and link quality prediction for $\mathrm{V} 2 \mathrm{~V}$ communication [8].

For the purpose of prediction of future best beam, we propose a novel framework where the received signals from each BS beam are used to construct a CC for each beam used by the BS. The beam prediction framework consists 

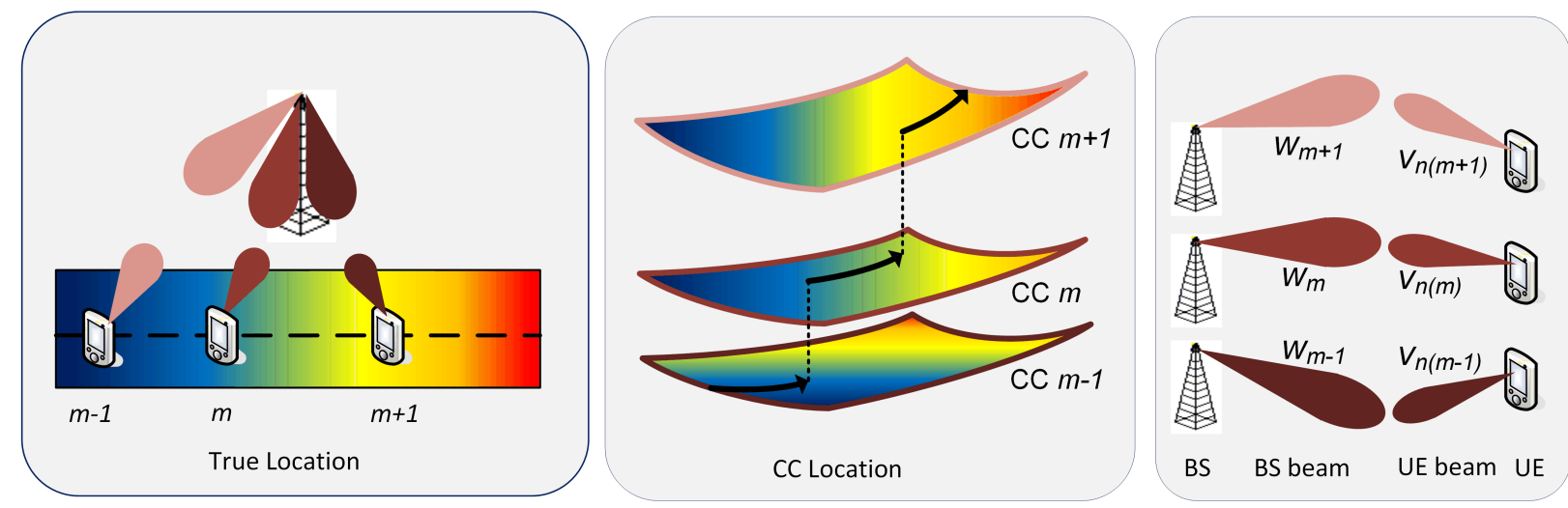

Fig. 1. Beam prediction based on channel charting. (Left); a cell (a street segment) served by a multi antenna BS. (Middle); per beam CCs constructed at the BS. (Right); BS beam and the corresponding best UE beam for different locations.

of two phases where the first phase involves collection of CSI information under each BS beam necessary to construct per BS beam CC at the BS. The second phase is the online usage phase, where the collected data is leveraged to perform prediction in real time. The real time prediction helps reduce the overhead and delay associated with the beam alignment in mmWave systems. In the entire prediction framework, we do not use any physical location information of the UEs but use only the received CSI at the BS.

The rest of the paper is organized as follows. Section II elaborates on the system model used as the basis for analysis and simulation. Section III expands on the concept of channel charting and beam prediction with CCs. Section IV presents the simulation results and Section $\mathrm{V}$ concludes the paper by presenting the inferences from the results.

\section{SYSTEM MODEL}

We consider a cell (a sector of a BS) in a TDD system, serving a set of $K$ UEs. The BS has $M$ antennas while each UE $u$ has $N$ antennas. The UEs use orthogonal pilots to transmit to the BS. Analog beamformers are used at the UE and BS sides to create beams using phase shifters. The BS has several RF chains and can accordingly simultaneously transmit and receive from multiple beams. Here, for simplicity, we assume there are $M$ RF chains.

The MIMO channel at subcarrier $s$ between UE $u$ and the BS is denoted as $\mathbf{H}_{u, s} \in \mathbb{C}^{M \times N}$. The BS and UE are capable of forming a large discrete set of beams. For purposes of beam management, a limited number of beams is considered at the $\mathrm{BS}$. We shall typically use $B=M \mathrm{BS}$ beams. The beamforming vectors at the BS are $\mathbf{w}_{m} \in \mathbb{C}^{M \times 1}$ for $m=1, \ldots, B$ where $B$ is the number of BS beams. The beamforming vectors at the UE are $\mathbf{v}_{n} \in \mathbb{C}^{N \times 1}$ for $n=1, \ldots, B_{u}$ where $B_{u}$ is the number of UE beams. We use $B_{u}=N$ UE beams.

Wideband beams are considered; i.e., one beam for all frequencies. The BS uses a Discrete Fourier Transform (DFT) based codebook of size $M$ and the UE uses a DFT codebook of size $N$. The codebook $\mathbf{C}=\left[\mathbf{c}_{0}, \ldots, \mathbf{c}_{L-1}\right]$ for the BS and $\mathrm{UE}$ is generated as:

$$
\mathbf{c}_{l}=\frac{1}{\sqrt{L}}\left[1, e^{j 2 \pi \frac{l}{L}}, e^{j 2 \pi \frac{2 l}{L}}, \ldots, e^{j 2 \pi \frac{(L-1) l}{L}}\right]^{T},
$$

where $L=M$ for BS codebook and $L=N$ for UE codebook and $l=0, \ldots, L-1$.

The received signal from UE $u$ in subcarrier $s$ at the BS using beam $\mathbf{w}_{m}$ when the UE transmits on beam $\mathbf{v}_{n}$ is

$$
y_{m, n}^{u, s}=\mathbf{w}_{m}^{H} \mathbf{H}_{u, s} \mathbf{v}_{n} x_{s}+n_{u}=h_{m, n}^{u, s} x+n_{u},
$$

where $h_{m, n}^{u, s}=\mathbf{w}_{m}^{H} \mathbf{H}_{u, s} \mathbf{v}_{n}$ denotes the effective channel coefficient for BS beam $m$ and UE beam $n, x$ is the transmitted symbol and $n_{u}$ is an additive white Gaussian noise. The BS can estimate this effective channel from a UE pilot transmission. The effective channel vector measured from all beams at the BS from a UE transmission using beam $\mathbf{v}_{n}$ is then:

$$
\mathbf{h}_{n}^{u, s}=\mathbf{W}^{H} \mathbf{H}_{u, s} \mathbf{v}_{n},
$$

where $\mathbf{W}=\left[\mathbf{w}_{1}, \ldots, \mathbf{w}_{B}\right]$ is the matrix of BS beamforming vectors. The $\mathrm{BS}$ can measure the effective channel from one pilot transmission of the UE, assuming that it has an RF chain for each beam. If the BS has less than $M$ RF-chains, multiple UE transmissions are needed for the BS to measure this. The best combination of BS \& UE wideband beams is

$$
\left\{m_{u}^{*}, n_{u}^{*}\right\}=\underset{n, m}{\operatorname{argmax}} \mathbb{E}\left\{\left|h_{m, n}^{u, s}\right|^{2}\right\},
$$

where the expectation is with respect to frequency samples.

For each BS beam there exists a best UE beam. The UE has a function

$$
\hat{n}=\hat{n}(m)=\underset{n}{\operatorname{argmax}} \mathbb{E}\left\{\left|h_{m, n}^{u, s}\right|^{2}\right\},
$$

for selecting the best UE-beam for transmission towards/reception from BS beam $\mathbf{w}_{m}$, given the wideband channel of the UE. The BS does not know which beam the UE uses, only which BS beam it is supposed to transmit towards and receive from. The channel vector between the $B$ BS beams and the UE baseband receiver thus is conditioned on 
the assumption of the UE regarding which beam the BS uses. If the UE assumes that the BS uses beam $m$, this channel vector becomes

$$
\mathbf{h}_{\hat{n}(m)}^{u, s}=\mathbf{W}^{H} \mathbf{H}_{u, s} \mathbf{v}_{\hat{n}(m)} .
$$

Accordingly, the BS cannot unilaterally measure and find the best beam towards the user. The BS can measure the elements in the vector (6) from UE transmissions, but the BS cannot measure what the channel coefficient at a beam $m^{\prime} \neq m$ would be, if the UE would select the best precoder $\mathbf{v}_{n\left(m^{\prime}\right)}$ for transmission towards $\mathbf{w}_{m^{\prime}}$. There is a beam-mismatch problem arising from UE autonomous precoding; the precoder $\mathbf{v}_{n\left(m^{\prime}\right)}$ may or may not be the same as $\mathbf{v}_{n(m)}$.

\section{BeAm PREDiction USING CHANNEL ChARTING}

To solve the beam mismatch problem, we apply ML based on a channel charting approach. Here, we first introduce the concepts related to channel charting and then proceed with an elaboration of the proposed beam prediction with CCs. The concept of beam prediction using beam-based CCs is illustrated in Fig. 1. The idea is to utilize per beam CCs of the best beam at the present time instant to predict the best beam in the next time instant for a mobile UE.

\section{A. Channel Charting}

The base assumption of channel charting is that the CSI covariance matrix of a location reflects the large-scale effects of the wireless channel. To create a $\mathrm{CC}$ for a given basestation, first the CSI covariance matrix $\boldsymbol{R}_{u}$ for the UE $u$ is processed to obtain channel features $\boldsymbol{f}_{u}$ that capture the largescale properties of the wireless channel [6].

The set of collected features $\left\{\boldsymbol{f}_{u}\right\}_{u=1}^{K}$ for the set of UEs $\mathcal{K}=\{1, \ldots, K\}$ seen by the BS are used to calculate the dissimilarity matrix $\boldsymbol{D} \in \mathbb{R}_{+}^{K \times K}$ which consists of the feature dissimilarities $d_{u, u^{\prime}}$ between pairs of UEs $u, u^{\prime} \in \mathcal{K}$. There are different ways to extract channel features and to obtain the dissimilarity matrix [3], [5]. In this paper, we directly calculate the dissimilarity matrix for a given base station from the covariance matrices $\left\{\boldsymbol{R}_{u}\right\}_{u=1}^{K}$ at the BS using the Collinearity Matrix Distance (CMD) metric [9]. The dissimilarity for two UEs $u$ and $u^{\prime}$ with covariances $\boldsymbol{R}_{u}$ and $\boldsymbol{R}_{u^{\prime}}$ under CMD measure is the Frobenius norm of normalized covariance matrices:

$$
d_{\mathrm{CMD}}\left(\boldsymbol{R}_{u}, \boldsymbol{R}_{u^{\prime}}\right)=1-\frac{\operatorname{Tr}\left(\boldsymbol{R}_{u} \boldsymbol{R}_{u^{\prime}}\right)}{\left\|\boldsymbol{R}_{u}\right\|_{\mathrm{F}}\left\|\boldsymbol{R}_{u^{\prime}}\right\|_{\mathrm{F}}}
$$

where $\operatorname{Tr}$ indicates trace operator.

Dimensionality reduction techniques like Isomaps [10] and t-Distributed Stochastic Neighbor Embedding (t-SNE) [11] are applied on the dissimilarity matrix to obtain a two-dimensional chart containing coordinates corresponding to each UE, referred to as its chart location. The chart location of each UE is such that the neighborhood relations of its physical location with other physical locations of other UEs within the cell are preserved in the chart. The quality of CCs is characterized in terms of Continuity (CT), Trustworthiness
(TW) and Kruskal Stress (KS) metrics [12]. These metrics quantify how well the $\mathrm{CC}$ locations preserve the distance relations of the true physical domain. CT represents how well the neighbors in physical domain are preserved in the $\mathrm{CC}$ domain. TW quantifies how well the $\mathrm{CC}$ refrains from introducing false neighbors when compared to the physical domain. KS indicates how well the CC preserves the global structure in the mapping. CT, TW and KS values lie between 0 and 1 . It is desirable to have CT and TW as close to 1 as possible and $\mathrm{KS}$ as close to 0 as possible.

\section{B. Beam Prediction Using CC}

Beam Prediction using $\mathrm{CC}$ is based on the channel reciprocity in TDD. For each RF-chain of the BS, the BS can measure one element of the vector (6) in each subframe where the UE is transmitting. If the BS has one RF-chain, and the $\mathrm{UE}$ transmits only in subframes where the BS receives from the indicated best beam of the UE, the BS gets only 1D channel information from the UE, which is not sufficient to create a CC. Essentially, the BS receives transmissions from an effective $1 \times 1$ SISO channel. If the UE transmits pilot information in multiple subframes, where the BS listens to multiple beams, or the BS has multiple RF-chains, the BS acquires a richer channel which can be used for channel charting.

If the BS measures using $M$ beams, the effective channel measured is $1 \times M$ SIMO. However, due to the beam misalignment problem, the measured channel vector depends on the BS beam $\mathbf{w}_{m}$ the UE is transmitting towards. At a given spatial location, the channel vectors $\mathbf{h}_{n(m)}^{u, s}$ and $\mathbf{h}_{n\left(m^{\prime}\right)}^{u, s}$ may differ. For a given $m$, this vector is changing continuously in space, but if we select different $n(m)$ at different spatial locations, the corresponding channels would not change continuously in space. To create reliable $\mathrm{CCs}$, we thus have to condition the $C C$ on the BS beam that the UE transmits towards. The BS thus constructs a separate $\mathrm{CC}$ for each BS beam, from the received signals (6) where the UEs at different locations transmit with the location specific $\mathbf{v}_{n(m)}$ which is the best beam at that location for transmitting towards $\mathbf{w}_{m}$.

At each sample location, there exists a best BS beam which is determined as:

$$
\hat{m}=\arg \max _{m} \mathbb{E}\left\{\left|h_{m, n(m)}^{u, s}\right|^{2}\right\} .
$$

We now annotate all the CCs with information about the best BS beam $\hat{m}$ at each sample point, which enables best BS beam prediction.

We consider a $\mathrm{CC}$ based beam prediction, that consists of two phases: The training (offline) and the prediction (online). In the training phase, the BS collects channel features for UEs transmitting towards different beams. Data is accumulated, and a BS-specific beam is constructed. There is a set of UEs selecting their respective best UE beam $\hat{n}(m)$ for transmission towards BS beam $\mathbf{w}_{m}$. Note that in the training phase, $\mathbf{w}_{m}$ may or may not be the best BS beam for a user in this set. The UEs transmit pilot transmissions towards the BS at multiple 
time instances. The covariance matrix of UE $u$ towards BS beam $m$ is then

$$
\mathbf{R}_{u, m}=\mathbb{E}\left\{\mathbf{h}_{\hat{n}(m)}^{u, s}\left(\mathbf{h}_{\hat{n}(m)}^{u, s}\right)^{H}\right\},
$$

where, the expectation is over frequency and spatial samples. The covariances of this set of UEs is used to create the CC of beam $m$. The CMD measure is used to obtain the dissimilarity between covariance matrices. The manifold learning techniques of Isomaps and t-SNE are used to create the CC from the dissimilarity matrices. The $\mathrm{CC}$ is annotated based on the best beam information collected.

In the prediction (online) phase, the CCs with associated best beam data are used to predict next position's best beam. Assume that a UE transmits with beam $\mathbf{v}_{n(m)}$, where $m$ may or may not indicate the best BS beam at the location of the UE. The BS measures the CSI feature for the UE, and obtains the $\mathrm{CC}$ coordinates using out-of-sample extension for the new UE positions on the CC of beam $m$. This can be done, e.g., by calculating the dissimilarities of the covariances of the new data point with those of the existing CC points and approximating the $\mathrm{CC}$ location of the new data point as that of the charted UE with the smallest dissimilarity with the new UE. From the annotations of the best beams on sample points, the BS can deduce the best BS beam index $\hat{m}$ for the location of the UE.

Furthermore, we can predict mobility of the UE, and the best beam at a future time instance. After obtaining the approximate $\mathrm{CC}$ locations for the past positions of the UE, we can predict the future chart position of the UE from its past positions. We consider simple linear prediction of future $(t+1)^{t h}$ CC position $\hat{\mathbf{z}}_{t+1}$ using the past two CC positions $\mathbf{z}_{t-1}$ and $\mathbf{z}_{t}$ which is given as:

$$
\hat{\mathbf{z}}_{t+1}=\mathbf{z}_{t}+\left(\mathbf{z}_{t}-\mathbf{z}_{t-1}\right) .
$$

The two point CC based prediction is illustrated in Fig. 2. Similarly, we can go for a more sophisticated position prediction methods considering more past positions than just two. We consider a 5-point prediction scheme where the points from index $t$ through $t-4$ are used to predict the $(t+1)^{t h}$ point. For this, we consider the approach that the predicted location is sum of the past value and a factor related to the difference of the sample times. Thus, if we consider the average of four such terms $\mathbf{z}_{t}+\left(\mathbf{z}_{t}-\mathbf{z}_{t-1}\right), \mathbf{z}_{t}+\frac{\left(\mathbf{z}_{t}-\mathbf{z}_{t-2}\right)}{2}, \mathbf{z}_{t}+\frac{\left(\mathbf{z}_{t}-\mathbf{z}_{t-3}\right)}{3}$, and $\mathbf{z}_{t}+\frac{\left(\mathbf{z}_{t}-\mathbf{z}_{t-4}\right)}{4}$, we arrive at future CC prediction

$$
\hat{\mathbf{z}}_{t+1}=\frac{73}{48} \mathbf{z}_{t}-\frac{1}{4}\left(\mathbf{z}_{t-1}+\frac{\mathbf{z}_{t-2}}{2}+\frac{\mathbf{z}_{t-3}}{3}+\frac{\mathbf{z}_{t-4}}{4}\right) .
$$

After predicting the future $\mathrm{CC}$ position using the above simple approaches, from the annotations of the sampling points of the CC for beam $m$, we can now predict the best beam of the UE at time $t+1$.

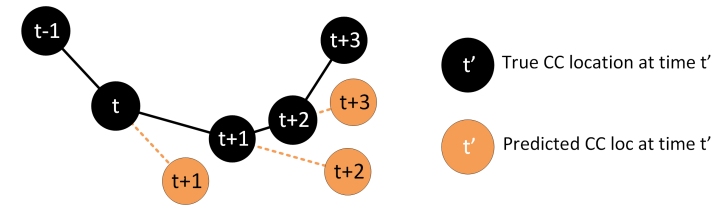

Fig. 2. Two-point prediction of CC locations.

\section{Beam Prediction Performance}

The prediction error is used as the performance measure of the $\mathrm{CC}$ based beam prediction computed as:

$$
\operatorname{Err}=\frac{\sum_{p=1}^{P} \mathbb{1}_{\hat{b}_{p} \neq b_{p}}}{P},
$$

where $P$ is the number of beam predicted locations, $\hat{b}_{p}$ is the predicted beam at location $p, b_{p}$ is the true best beam at CC location $b$ and $\mathbb{1}$ is the indicator function. The physical location for beam management is considered in [4], [5]. Therefore, the error performance of the $\mathrm{CC}$ based beam predictor is compared with error performance of beam prediction using physical locations of the UEs. For this, in the offline phase, the physical location is annotated with the best beam information and in the online phase, the UE future physical location is predicted based on previous UE physical locations and the best beam is determined from the annotated physical location chart. To determine the error performance of prediction using physical locations, we first predict the future position of the UE using true locations of its past positions in the same way as for prediction with CCs. Then, the best beam for the predicted position is assigned based on the nearest UE location among the UEs used for CC creation in the physical domain.

\section{Simulation Results}

\section{A. Simulation Settings}

The Quasi Deterministic Radio Channel Generator (QuaDRiGa) is used to simulate the radio environment and obtain the CSIs of the wireless links [13]. We consider a single cell setting with a BS at the $[-114-100]$ at an approximate distance of $151 \mathrm{~m}$ from the street segment containing UEs. The simulation settings in Table I are used to generate the CSI information from QuaDRiGa.

TABLE I

Simulation Parameters

\begin{tabular}{llll}
\hline Parameter & Value & Parameter & Value \\
\hline Center Freq. & $28 \mathrm{GHz}$ & Subcarriers & 256 \\
Subcarrier BW. & $240 \mathrm{KHz}$ & UE speed & $50 \mathrm{Kmph}$ \\
BS Array & $32 \mathrm{ULA}$ & UE Array & $8 \mathrm{ULA}$ \\
Scenario & $3 \mathrm{GPP} 38.901$ & BS height & $25 \mathrm{~m}$ \\
& UMa-NLOS & & \\
\hline
\end{tabular}

We consider a street segment of $10 \mathrm{~m} \times 10 \mathrm{~m}$ between coordinates $\left[\begin{array}{ll}0 & 10\end{array}\right]$ on the $\mathrm{x}$-axis and $\left[\begin{array}{ll}0 & 10\end{array}\right]$ on the $\mathrm{y}$-axis. The direction of motion is considered along the positive $\mathrm{x}$ direction for the collection of the spatial samples used for 

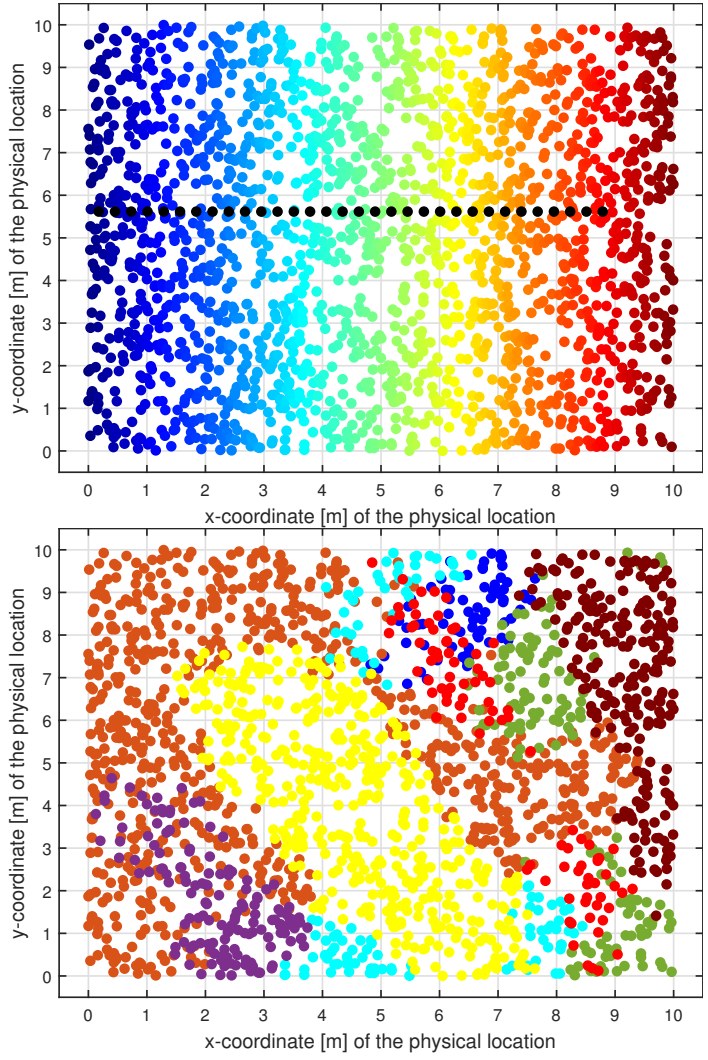

Fig. 3. (Top); Sampled UE physical locations marked by colors with a sampled mobility path. (Bottom); Best beam information as a function of the physical location with different best beams indicated by different colors. The best beam in the central region, colored with yellow, is beam 23

covariance calculation and 2000 UEs are uniformly distributed in this street segment for the purpose of constructing the CCs. We assume a UE pilot transmission every $1 \mathrm{~ms}$, and the data from 100 such transmissions is collected for each UE. A covariance matrix estimate is created from the channel estimates of these 100 transmissions over a distance of 1.39 $m$ with the considered UE speed. We constructed CCs using Isomaps and t-SNE techniques. For each of the UEs, the best BS serving beam is determined during the training phase and annotated to their CC location.

Then, 35 separate mobile UEs moving in the street segment along the x-direction are considered. For each of these UEs, we consider a path of length of about $10 \mathrm{~m}$. The starting points of the paths lie uniformly distributed between [ $\left[\begin{array}{ll}0 & 0.28\end{array}\right]$ $\mathrm{m}$ on $\mathrm{x}$-axis and $\left[\begin{array}{ll}0 & 10\end{array}\right] \mathrm{m}$ on $\mathrm{y}$-axis. Along this path, we will again collect CSI data for the UE for every $1 \mathrm{~ms}$. The channel covariance data is calculated for every $20 \mathrm{~ms}$. We have considered 100 samples for each covariance calculation. Thus for every $20 \mathrm{~ms}$, we obtain a sample covariance considering the subsequent 100 channel samples. This kind of sampling provides us with slowly varying covariances. The relative physical locations of the UE path points are shown in the top of Fig. 3. A single UE's mobility path is marked in the middle of the street as an illustration. The best beam for each UE location in the physical domain is shown on the bottom of Fig. 3. A mobility path of a mobile UE in the middle of
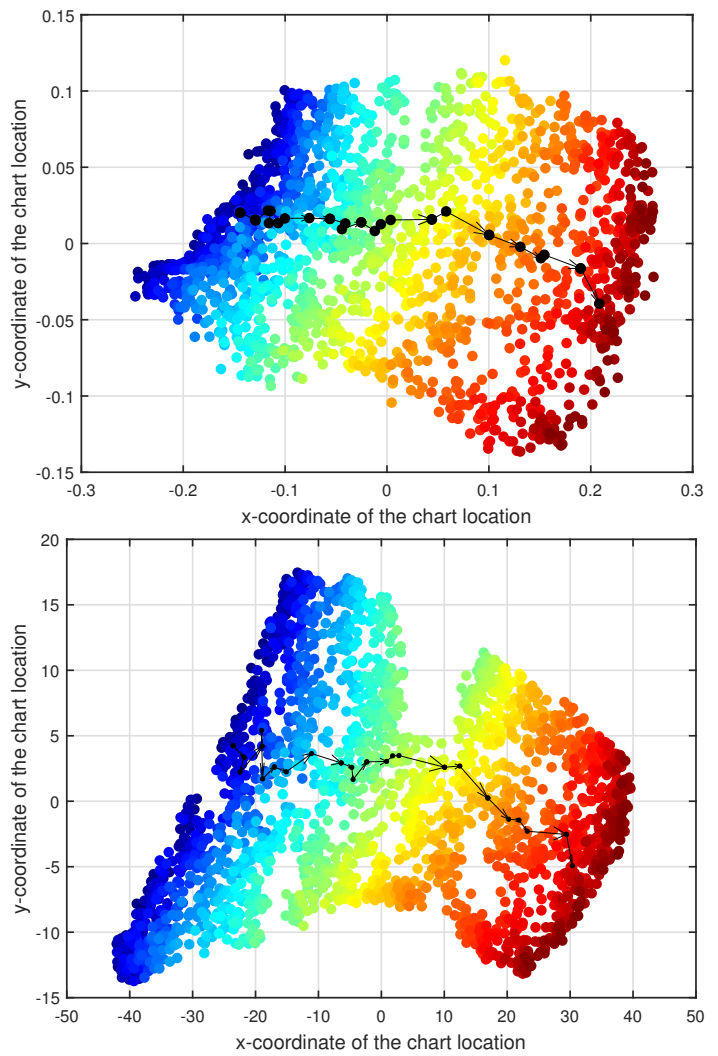

Fig. 4. CCs for beam 23 and the chart locations of a single mobility path from Fig. 3 marked: (Top); Isomap CC. (Bottom); t-SNE CC.

the street is shown in the Isomap CC and t-SNE CC for beam 23 in Fig. 4. The CCs show the approximative structure of the actual physical layout of the UEs which can be observed in the overall square shape and relative locations indicated by the colormap. It can also be noted that even an approximated mobility path on the $\mathrm{CC}$ has a smooth progression. The Isomap $\mathrm{CC}$ and t-SNE CC for beam 23 annotated with best beam information are shown on the top and bottom parts of Fig. 5 .

The performance metrics of the Isomap and t-SNE CCs are tabulated in Table II for the dominant beams in the region considered; beams 19 and 23. The high values close to 1 for the quality scores $\mathrm{CT}$ and TW indicates that the CCs obtained are high-quality in terms of maintaining neighborhood relations of the UEs in the physical dimension.

TABLE II

CC Performance Measures

\begin{tabular}{c|cccc}
\hline \multirow{2}{*}{$\begin{array}{c}\text { Performance } \\
\text { Measures }\end{array}$} & \multicolumn{2}{|c}{ Isomaps } & \multicolumn{2}{c}{ t-SNE } \\
\cline { 2 - 5 } & Beam 19 & Beam 23 & Beam 19 & Beam 23 \\
\hline TW & 0.977 & 0.985 & 0.981 & 0.986 \\
CT & 0.982 & 0.989 & 0.980 & 0.988 \\
KS & 0.308 & 0.300 & 0.326 & 0.316 \\
\hline
\end{tabular}

\section{B. Beam Prediction Performance and Discussion}

The beam prediction accuracies for the Isomap and t-SNE CCs are tabulated in the Table. III. Maximum prediction accu- 

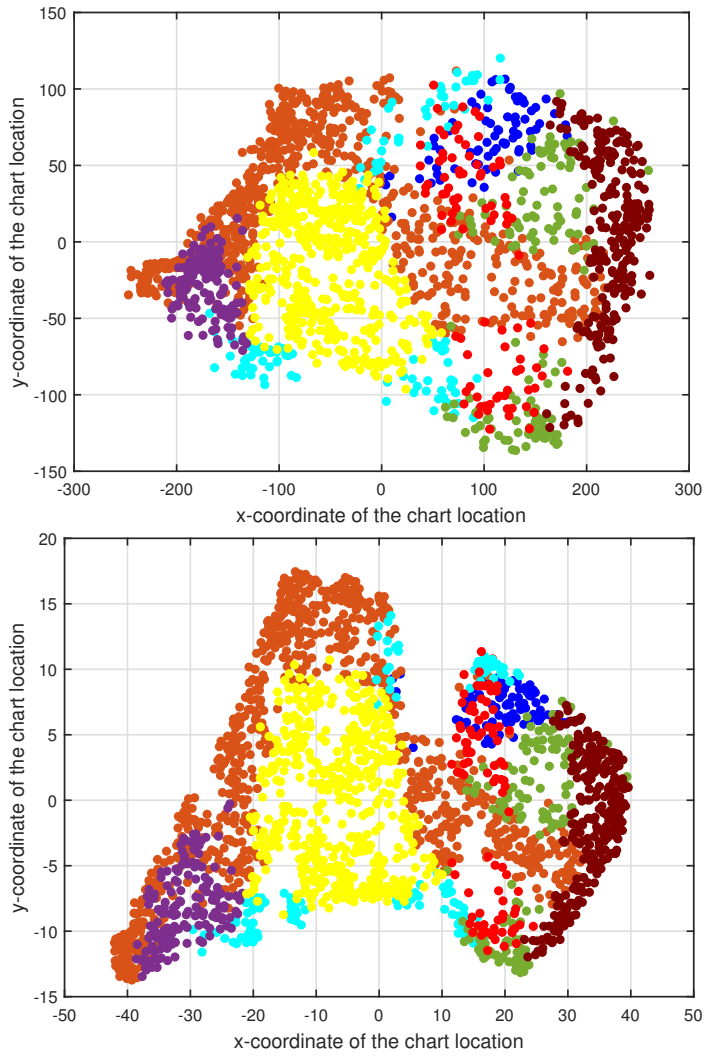

Fig. 5. CCs for beam 23 annotated with best beam information: (Top); Isomap CC. (Bottom); t-SNE CC.

TABLE III

BEAM PREDICTION ACCURACY

\begin{tabular}{ccc}
\hline Physical & Isomaps & $\boldsymbol{t}$-SNE \\
\hline 81.1 & 68.5 & 70.8 \\
79.6 & 70.2 & 70.4 \\
\hline
\end{tabular}

racy of around $71 \%$ has been achieved. The maximum beam prediction accuracy for physical location based prediction is $81 \%$. There is a performance loss of the CC-based prediction as compared to physical location based prediction. This is the cost from relying on existing Radio Access Network internal CSI for localization, instead of using GPS information.

\section{Conclusions}

The challenge of beam alignment in mmWave communication system can be tackled by using prediction of future best beams. CC can help deal with the beam misalignment problem. A CC based framework to perform such predictions was considered. Simulations were conducted to evaluate the performance of the prediction scheme. Prediction accuracies achieved using CC formed with only CSI information of the UEs were slightly worse compared to the prediction accuracies using physical location of the UEs. In the proposed framework, we do not require use of actual physical locations of the UEs. This demonstrates the potential of using channel charting in performing proactive RRM. There are some areas of improvement that can help improve the performance of the prediction such as using neural networks based prediction.

\section{REFERENCES}

[1] M. Giordani, M. Polese, A. Roy, D. Castor, and M. Zorzi, "A tutorial on beam management for 3GPP NR at mmWave frequencies," IEEE Communications Surveys Tutorials, vol. 21, no. 1, pp. 173-196, 2019.

[2] Y. R. Li, B. Gao, X. Zhang, and K. Huang, "Beam management in Millimeter-Wave communications for 5G and beyond," IEEE Access, vol. 8, pp. 13282-13293, 2020.

[3] Y. Wang, A. Klautau, M. Ribero, A. C. K. Soong, and R. W. Heath, "MmWave Vehicular Beam Selection With Situational Awareness Using Machine Learning," IEEE Access, vol. 7, pp. 87479-87 493, 2019.

[4] M. Arvinte, M. Tavares, and D. Samardzija, "Beam management in 5G NR using geolocation side information," in Proc. of 53rd Annual Conference on Information Sciences and Systems (CISS), 2019, pp. 16.

[5] Y. Heng and J. G. Andrews, "Machine Learning-Assisted Beam Alignment for mmWave Systems," in Proc. of IEEE Global Communications Conference (GLOBECOM), Dec. 2019, pp. 1-6, iSSN: 2576-6813.

[6] C. Studer, S. Medjkouh, E. Gonultaş, T. Goldstein, and O. Tirkkonen, "Channel Charting: Locating Users Within the Radio Environment Using Channel State Information," IEEE Access, vol. 6, pp. 47682-47698, 2018.

[7] J. Deng, S. Medjkouh, N. Malm, O. Tirkkonen, and C. Studer, "Multipoint Channel Charting for Wireless Networks," in Proc. of 52nd Asilomar Conference on Signals, Systems, and Computers, Oct. 2018, pp. 286-290.

[8] H. Al-Tous, T. Ponnada, C. Studer, and O. Tirkkonen, "Multipoint channel charting-based radio resource management for $\mathrm{V} 2 \mathrm{~V}$ communications," EURASIP Journal on Wireless Communications and Networking, vol. 2020, no. 1, p. 132, Jun. 2020.

[9] M. Kurras, S. Dai, S. Jaeckel, and L. Thiele, "Evaluation of the Spatial Consistency Feature in the 3GPP Geometry-Based Stochastic Channel Model," in Proc. of the IEEE Wireless Communications and Networking Conference (WCNC), Apr. 2019, pp. 1-6.

[10] J. B. Tenenbaum, V. de Silva, and J. C. Langford, "A Global Geometric Framework for Nonlinear Dimensionality Reduction," Science, vol. 290, no. 5500, pp. 2319-2323, 2000.

[11] L. van der Maaten and G. Hinton, "Visualizing data using tSNE," Journal of Machine Learning Research, vol. 9, pp. 2579-2605, Nov. 2008.

[12] L. Maaten, E. Postma, and H. Herik, "Dimensionality reduction: a comparative review," Journal of Machine Learning Research, vol. 10, pp. 66-71, 2009.

[13] S. Jaeckel, L. Raschkowski, K. Borner, and L. Thiele, "QuaDRiGa: A 3D Multi-Cell Channel Model With Time Evolution for Enabling Virtual Field Trials," IEEE Trans. Antennas Propag., vol. 62, no. 6, pp. 32423256, Jun. 2014. 